DOI: $10.24850 /$ j-tyca-2021-01-10

Notas

\title{
Disposición a pagar para mejorar la calidad del agua en Zapopan
}

\section{Willingness to pay to improve water quality in Zapopan}

Hugo Briseño1, ORCID: https://orcid.org/0000-0001-8465-8683

Edith Macedo2, ORCID: https://orcid.org/0000-0003-1639-6006

1Universidad Panamericana. Facultad de Ciencias Económicas y Empresariales, Álvaro del Portillo 49, Zapopan, Jalisco, 45010, México, hbriseno@up.edu.mx

2Universidad Panamericana. Facultad de Ciencias Económicas y Empresariales. Álvaro del Portillo 49, Zapopan, Jalisco, 45010, México, emacedo@up.edu.mx

Autor para correspondencia: Hugo Briseño, hbriseno@up.edu.mx

\section{Resumen}

El objetivo que se persigue con el presente artículo es explorar los factores que están asociados con la disposición a pagar para mejorar la calidad del 
agua en la ciudad mexicana de Zapopan, Jalisco. Se lleva a cabo una encuesta a 400 hogares, la cual arroja que los consultados toman agua embotellada (99\%); consideran que el agua suministrada huele mal (53 $\%)$ y que está contaminada (69\%); temen por su salud o la de sus familiares por beber del grifo (74 \%), pero estarían dispuestos a tomar agua del mismo si la calidad mejorara (77\%). Sin embargo, más de la mitad no estaría dispuesta a pagar más por mejorar la calidad del agua o pagaría no más de 40 pesos (31 y $22 \%$, respectivamente). A través de un modelo logit ordenado se encuentra que existe una relación significativa y positiva entre la disposición a pagar por mejorar la calidad del agua y estar abierto a tomar agua del grifo si esto sucede; a percibir que las autoridades encargadas del sector agua hacen lo correcto; a una creencia de escasez de agua en la colonia; a tener personas con problemas de salud en casa, y al nivel de ingresos. Por otro lado, mayor disposición de pago se relaciona negativa y significativamente con la percepción de que el agua huela mal, el nivel de confianza en los otros y la edad.

Palabras clave: disposición a pagar, agua de calidad, servicio de agua, suministro de agua, tarifa de agua.

\section{Abstract}

The objective of this article is to explore the factors that are associated with the willingness to pay (WTP) to improve water quality in the Mexican city of Zapopan, Jalisco. A survey of four hundred households is carried 
out. It shows that the respondents drink bottled water (99\%); consider that the supplied water smells bad (53\%) and that it is contaminated (69 $\%)$; they fear for their health or that of their family members from drinking from the tap (74 \%), but would to drink water from it if the quality improved (77 \%). However, more than half would not be willing to pay for an improvement in water quality or pay up to a limit of 40 pesos (31 and $22 \%$, respectively). Through an orderly logit model, it is found that there is a significant and positive relationship between the willingness to pay for improving water quality and being open to drinking tap water if it happens, perceiving the authorities responsible for water sector do the right actions; a belief of water scarcity in the colony; having people with health problems at home, and the income level. On the other hand, more willingness to pay relates negatively and significantly with the perception that water smells bad, the level of trust in others, and age.

Keywords: willingness to pay, safe drinking water, water service, water quality, water supply, pricing water.

Recibido: 08/02/2020

Aceptado: $15 / 06 / 2020$

\section{Introducción}


Aunque los organismos operadores de agua en México se empeñan porque la calidad del agua que llega a los hogares sea apta para el consumo humano, la realidad es que muchas viviendas optan por beber el líquido embotellado. Sin embargo, el uso de plásticos para generar botellas y garrafones de agua no es amigable con el ambiente, y adquirir soluciones para purificar y tratar el agua en casa no son económicamente convenientes (Friedler \& Hadari, 2006). Además, el agua embotellada es mucho más cara que el agua de la llave. La tarifa por metro cúbico más onerosa para un consumo de 30 metros cúbicos al mes en el año 2015, correspondiente a la ciudad de Querétaro, Querétaro, fue de 20.8 pesos; aunque hay ciudades como Zapopan, Jalisco, en que la tarifa fue de 11.3, y como Tlaxcala, Tlaxcala, que fue de 5.6 (Conagua, 2016). Una botella de agua de litro, dependiendo dónde se adquiera y la marca, puede superar los 10 pesos.

Del mismo modo que muchas otras ciudades en México, el actual sistema municipal de suministro de agua en Zapopan encara una serie de desafíos. Entre éstos destacan la falta de recursos económicos suficientes para mantener en buenas condiciones técnicas la red de distribución de agua potable y que ésta llegue a la población a los menores costos posibles con niveles adecuados de calidad (Briseño \& Sánchez, 2018). Además, el agua potable en las ciudades de México a menudo está 
contaminada y no es confiable para beber (Vásquez, Mozumder, Hernández-Arce, \& Berrens, 2009).

Aunque existen diferentes esquemas tarifarios en México, poco se ha estudiado sobre la disponibilidad de pago de los ciudadanos para mejorar la calidad del agua suministrada por los organismos operadores.

El objetivo que se persigue con este trabajo es conocer cuáles son los factores que se asocian con la disposición a pagar para mejorar la calidad del agua en el municipio mexicano de Zapopan, Jalisco. En el presente artículo se realiza una revisión de estudios previos sobre disposición a pagar para mejorar la calidad del agua; se lleva a cabo una contextualización del municipio de Zapopan; se describe la metodología empleada; se realiza estadística descriptiva de los datos; se lleva a cabo un modelo econométrico para conocer los factores de impacto en la disposición de pago, y se ofrecen algunas conclusiones.

\section{Revisión de la literatura}

Prácticamente todos los estudios que exploran los factores asociados con la disposición a pagar para mejorar la calidad del agua utilizan el método 
de valoración contingente (MVC), mismo que se describirá más adelante. En este apartado se revisan los principales hallazgos que se encuentran en la literatura.

Polyzou, Jones, Evangelinos y Halvadakis (2011) realizan una encuesta en la ciudad griega de Mitilene, y encuentran relación significativa y positiva entre la disposición a pagar más por la calidad del agua e indicadores relacionados con el capital social, así como con el ingreso.

Tanellari, Bosch, Boyle y Mykerezi (2015), en su estudio sobre la disposición a pagar para mejorar la calidad e infraestructura del agua en el norte de Virginia y los suburbios de Washington, EUA, encuentran como principal aportación que existe una relación significativa negativa entre la disposición a pagary el costo de las alternativas planteadas para mejorar la calidad del agua.

La fuente de suministro de agua también es un aspecto relevante. Tussupova, Berndtsson, Bramryd y Beisenova (2015), en su estudio sobre disposición a pagar por mejorar el suministro de agua en Kazajistán, muestran evidencia de que aquellos cuya fuente de abastecimiento proviene del subsuelo están dispuestos a pagar menos, pues perciben que es de buena calidad.

El único estudio que se detectó para México fue el del caso de Parral, Chihuahua. Vásquez, Mozumder, Hernández-Arce y Berrens (2009) Ilevan a cabo una encuesta cuyos resultados -analizados con un modelo econométrico-concluyen que hay una relación significativa positiva entre 
la disposición a pagar por mejorar la calidad del agua y las variables educación, ingreso, confiabilidad de que el sistema mejorado dotará de agua de calidad y la percepción de que sí es posible que se lleve a cabo el proyecto de mejora; y una relación significativa negativa con la edad, la percepción de calidad y la presencia de alguien escuchando mientras se llevaba a cabo la encuesta.

Quizás el estudio más relevante que se ha efectuado en los últimos años es hecho en la ciudad de Jacksonville, Florida, EUA. Chatterjee, Triplett, Johnson y Ahmed (2017) aplican una encuesta y, a través de un modelo logit ordenado, encuentran que la disposición a pagar por mejorar la calidad del agua tiene una relación significativa positiva con la preocupación por enfermarse, la presencia de niños en el hogar y el nivel de educación; así como una asociación significativa negativa con la creencia de que el agua no huele mal, saber que el gobierno conoce la situación de la mala calidad del agua del grifo, la desconfianza en las autoridades y la edad.

En la Tabla 1 se muestra una síntesis de los factores asociados con la disposición a pagar por la calidad del agua que se encontraron en la revisión de literatura.

Tabla 1. Factores de disposición a pagar por la calidad del agua. Fuente: elaboración propia. 


\begin{tabular}{|l|l|}
\hline \multicolumn{1}{|c|}{ Relacionados positivamente } & Relacionados negativamente \\
\hline Ingreso & $\begin{array}{l}\text { Costo de la solución } \\
\text { Fuente subsuelo (percepción de } \\
\text { buena calidad) }\end{array}$ \\
Capital social & Edad \\
Confianza en la autoridad & Percepción de calidad \\
Fuente cielo abierto (percepción de & Percepción de que el agua no tiene \\
mala calidad) & mal olor \\
Preocupación por enfermarse & Percibir que el gobierno conoce la \\
Presencia de niños & situación de mala calidad del agua \\
& Desconfianza en la autoridad \\
\hline
\end{tabular}

\section{Contexto de la ciudad de Zapopan}

La ciudad de Zapopan es la segunda más importante de la Zona Metropolitana de Guadalajara (ZMG), México, con una población estimada de 1332272 personas de acuerdo con información de la encuesta 
intercensal del INEGI en el año 2015. Zapopan es el municipio más grande en extensión de la ZMG y cuenta con una interesante composición sociocultural, con viviendas en el sector económico más alto, que contrastan con población en situación de pobreza o vulnerabilidad. De acuerdo con el Instituto de Información Estadística y Geográfica (IIEG) (2015), la población pobre o vulnerable del municipio de Zapopan alcanzó 28.1 y $33.6 \%$, respectivamente.

En México, el principal organismo y autoridad del servicio público del agua es la Comisión Nacional del Agua (Conagua), responsable de asignar el recurso para diferentes usos, entre los que se incluyen los públicos urbanos.

El Sistema Intermunicipal de los Servicios de Agua Potable y Alcantarillado (SIAPA) es un organismo público descentralizado de la administración del estado de Jalisco, y es el encargado de suministrar del líquido a la ciudad de Zapopan, además de las ciudades de Guadalajara, Tlaquepaque y Tonalá.

El suministro de agua a la ZMG se da a través de tres principales fuentes: lago de Chapala, por un acueducto (61\%); pozos subterráneos (27\%), y presa de Calderón (12 \%) (CEA, 2014). Sin embargo, uno de los grandes problemas que se enfrenta la ZMG en la gestión, administración y distribución del agua es la contaminación y escasez del recurso, lo que no garantiza que las fuentes de abastecimiento sean sostenibles (Torres-Rodríguez, 2013). Los niveles de contaminación de las cuencas hidráulicas de la ZMG han permanecido constantes durante 
mucho tiempo, y son resultado de descargas agrícolas, industriales y urbanas (Ramírez, 2018). Además, el tratamiento del agua de la ciudad es insuficiente y no cubre a todos los contaminantes que se generan en la urbe (Camps, 2017).

Durante 2015, en Zapopan, la tarifa de agua para uso urbano promedio por metro cúbico para un consumo de 30 metros cúbicos fue de 11.3 pesos (Conagua, 2016). Según la Conagua (2016), esta tarifa se componía de 1.3 pesos de cargo fijo y 10 pesos de la cuota de agua menos el cargo fijo (Conagua, 2016). Sin embargo, dada la existencia de un subsidio cruzado, las tarifas comercial e industrial se ubicaron en 19.3 y 20 pesos, respectivamente (Conagua, 2016).

Aunque según lo estipulado en el estado de Jalisco, el SIAPA realiza análisis que permiten monitorear la calidad del agua en las plantas de tratamiento de agua de conformidad con la Norma Oficial Mexicana NOM127-SSA1-1994, la confianza de la población con el líquido proveniente del grifo ha sido desincentivada culturalmente por el consumo de agua potable por medio de botellas. Sin embargo, hasta la fecha, no se ha hecho ningún estudio para comprender las actitudes de los ciudadanos con respecto a la calidad del agua y su disposición a pagar por este servicio. De acuerdo con el Módulo de Hogares y Medio Ambiente (MOHOMA), los hogares mexicanos en 2017 gastaban una media de 52 pesos a la semana en la compra de agua embotellada o garrafones. Sin embargo, la representatividad de este módulo es sólo en nivel nacional. 
En este trabajo se realiza una encuesta a los habitantes de la ciudad de Zapopan, con el propósito de conocer sus percepciones sobre el suministro de agua en el municipio y su disposición a pagar por mejorar la calidad del líquido.

\section{Encuesta, metodología y estadística descriptiva}

El método de la valoración contingente (MVC) se utiliza en este estudio para diseñar una encuesta y estimar la disposición a pagar de los usuarios para mejorar la calidad del agua que es suministrada a los hogares. La implementación de encuestas bajo el método MVC se hace para recolectar información sobre preferencias acerca de la provisión de bienes y servicios públicos en los países en desarrollo (Whittington, Briscoe, Xinming, \& Barron, 1990).

Existen algunos estudios que proveen ejemplos de la aplicación de encuestas MVC en países en desarrollo, como los de Genius y Tsagarakis (2006), en Grecia; Pattanayak, Yang, Whittington y Bal-Kumar (2005), en Nepal; Rosado, Cunha-E-Sá, Ducla-Soares y Nunes (2006), en áreas urbanas de Brasil, y Vásquez et al. (2009), en Chihuahua, México. 
Bajo el método MVC se les pregunta a las personas su disposición a pagar por recibir un bien o servicio. Esta metodología enfatiza las preferencias declaradas de los encuestados en contraste con aquellos enfoques que usan las preferencias reveladas (Chatterjee et al., 2017). Sin embargo, obtener las preferencias de los encuestados bajo este método requiere un cuidadoso diseño del cuestionario, elección del modo de encuesta y la selección de la muestra (Bateman et al., 2002; Whittington, 2002).

En este artículo se realizan preguntas cerradas y se usa una encuesta cara a cara, a fin de estimar la disposición a pagar para mejorar la calidad del agua en la ciudad de Zapopan, Jalisco. Se ha demostrado que las preguntas cerradas son compatibles con incentivos y se han convertido en el enfoque estándar para simular la disposición a pagar (Cameron \& James, 1987); Chatterjee et al., 2017; Smith, 2006). En este tipo de cuestionamientos, los encuestados responden si él o ella podrían pagar más por recibir un beneficio determinado, y en qué magnitud.

En esta investigación se aplicó un cuestionario a 400 hogares representativos de Zapopan, en modalidad cara a cara durante octubre del año 2018. El municipio de Zapopan cuenta con un total de 277657 hogares de acuerdo con información del Instituto de Información Estadística y Geográfica del Estado de Jalisco, con un margen de error del $5 \%$ y con un nivel de confianza del $95 \%$; el tamaño de muestra significativo da un total de por lo menos 384 hogares. Así, se considera que 400 hogares es una muestra adecuada. El cuestionario se aplicó en 
20 colonias del municipio de Zapopan, que abarcan diferentes niveles socioeconómicos. De los 400 hogares encuestados, 120 (30 \%) corresponden a los estratos ABC+ (clase media alta y alta); 140 (35\%), al estrato C (clase media), y 140 (35\%) son del estrato D+ (clase media baja). El entrevistador determinó dichos estratos según la escala de la Asociación Mexicana de Agencias de Inteligencia de Mercado y Opinión (AMAI).

La encuesta tiene 20 reactivos asociados con el objetivo del presente trabajo. Los primeros cinco son acerca de las preferencias respecto al consumo de agua. Los siguientes siete abordan la percepción de la calidad y cantidad del agua, así como de la administración de la misma. Después, ocho preguntas son acerca de las características de la casa y del entrevistado.

La definición y estadística descriptiva de las principales variables utilizadas en el análisis se presentan en la Tabla 2. Estas variables son las siguientes: disposición a pagar (DISPAGAR); agua embotellada (EMBOTELLADA); propensión a tomar agua de la llave si la calidad mejora (LLAVESIMEJORA); agua de la llave huele mal (MALOLOR); agua contaminada (CONTAMINADA); preocupación por enfermedad proveniente de tomar agua de la llave (ENFERMARSE); las autoridades del sector agua hacen lo correcto (AUTBIEN); percepción de escasez (ESCASEZ); confianza en los demás (CONFIANZA); personas con mala salud (ENFERMOS); número de personas en el hogar (HABITANTES); propietario de la vivienda (CASAPROPIA); nivel de escolaridad (EDU); 
ingreso mensual promedio (INGRESO), y edad del encuestado (EDAD). Como se menciona al pie de la Tabla 2, fue necesaria la conversión de escala de algunas variables, dado que en ciertos niveles había muy pocas respuestas (ver pie de Tabla 2 ).

Tabla 2. Variables y estadística descriptiva. Fuente: elaboración propia.

\begin{tabular}{|c|c|c|c|c|c|c|}
\hline Variable & Descripción & Obs. & Media & $\begin{array}{l}\text { Desv. } \\
\text { est. }\end{array}$ & Mín. & Máx. \\
\hline DISPAGAR & $\begin{array}{l}\text { ¿Qué tanto aumento en su } \\
\text { factura mensual de agua } \\
\text { (en pesos mexicanos) } \\
\text { estaría dispuesto a pagar } \\
\text { para mejorar la calidad del } \\
\text { agua al grado de poder } \\
\text { beberla de la llave? (1. } \$ 0 ; \\
2 \text {. Hasta } \$ 40 ; 3 . \text { De } \$ 41 \text { a } \\
\$ 60 ; 4 . \text { De } \$ 61 \text { a } \$ 100 ; 5 \text {. } \\
\$ 101 \text { a } \$ 200 ; 6 . \text { De } \$ 201 \text { a } \\
\$ 299 ; 7 . \$ 300 \text { o más) }\end{array}$ & 400 & 2.69 & 1.63 & 1 & 7 \\
\hline EMBOTELLADA & $\begin{array}{l}1 \text { si toma agua embotellada; } \\
0 \text { en otro caso }\end{array}$ & 400 & 0.99 & 0.07 & 0 & 1 \\
\hline LLAVESIMEJORA & $\begin{array}{l}\text { Si la calidad del agua de la } \\
\text { llave fuera mejorada } \\
\text { ¿Estarías dispuesto a beber } \\
\text { de ésta? } \\
\text { (1. Sí; } 0 . \text { No) }\end{array}$ & 399 & 0.77 & 0.42 & 0 & 1 \\
\hline
\end{tabular}


Tecnología y

Ciencias $\approx$ Agua
2021, Instituto Mexicano de Tecnología del Agua

Open Access bajo la licencia CC BY-NC-SA 4.0

(https://creativecommons.org/licenses/by-nc-sa/4.0/)

\begin{tabular}{|c|c|c|c|c|c|c|}
\hline MALOLOR* & $\begin{array}{l}\text { ¿Qué tanto estás de acuerdo } \\
\text { en que el agua de la llave } \\
\text { huele mal? ( } 1 . \text { Desacuerdo; } \\
\text { 2. Ni de acuerdo ni } \\
\text { desacuerdo; 3. De acuerdo) }\end{array}$ & 400 & 2.13 & 0.95 & 1 & 3 \\
\hline CONTAMINADA* & $\begin{array}{l}\text { ¿Qué tan de acuerdo estás } \\
\text { en que el agua de la llave } \\
\text { está contaminada? } \\
\text { (1. Desacuerdo; } 2 \text {. Ni de } \\
\text { acuerdo ni desacuerdo; } 3 . \\
\text { De acuerdo) }\end{array}$ & 400 & 2.44 & 0.86 & 1 & 3 \\
\hline ENFERMARSE* & $\begin{array}{l}\text { Usando una escala de } 1 \text { a } 3 \\
\text { donde } 1 \text { significa "no estoy } \\
\text { preocupado" y } 3 \text { "estoy muy } \\
\text { preocupado", ¿Qué tan } \\
\text { preocupado estás tú o algún } \\
\text { miembro de tu familia de } \\
\text { enfermarse por beber agua } \\
\text { de la llave? }\end{array}$ & 400 & 2.56 & 0.76 & 1 & 3 \\
\hline AUTBIEN** & $\begin{array}{l}\text { Las autoridades } \\
\text { responsables del suministro } \\
\text { y calidad del agua hacen lo } \\
\text { correcto: (1. Nunca; } 2 . \\
\text { Algunas veces; } 3 \text {. La } \\
\text { mayoría del tiempo o } \\
\text { siempre) }\end{array}$ & 400 & 1.95 & 0.68 & 1 & 3 \\
\hline ESCASEZ* & $\begin{array}{l}\text { ¿Qué tan de acuerdo estás } \\
\text { en que existe un problema }\end{array}$ & 400 & 1.75 & 0.94 & 1 & 3 \\
\hline
\end{tabular}


Tecnología y

Ciencias $\cong$ Agua
2021, Instituto Mexicano de Tecnología del Agua

Open Access bajo la licencia CC BY-NC-SA 4.0

(https://creativecommons.org/licenses/by-nc-sa/4.0/)

\begin{tabular}{|c|c|c|c|c|c|c|}
\hline & $\begin{array}{l}\text { de escasez de agua en tu } \\
\text { colonia? (1. Desacuerdo; } 2 . \\
\text { Ni de acuerdo ni } \\
\text { desacuerdo; 3. De acuerdo) }\end{array}$ & & & & & \\
\hline CONFIANZA $* * *$ & $\begin{array}{l}\text { Siendo } 3 \text { "debe confiar } \\
\text { totalmente" y } 1 \text { "no debe } \\
\text { confiar" ¿Qué tanto puede la } \\
\text { gente confiar en los demás? }\end{array}$ & 400 & 1.99 & 1.00 & 1 & 3 \\
\hline ENFERMOS & $\begin{array}{l}\text { Incluyéndote a ti mismo, } \\
\text { ¿cuántas personas que } \\
\text { viven en tu hogar tienen } \\
\text { mala salud? ( } 1 ; 2 ; 3 ; 4 ; 5 \text { o } \\
\text { más) }\end{array}$ & 400 & 0.29 & 0.62 & 0 & 4 \\
\hline HABITANTES & $\begin{array}{l}\text { Incluyéndote a ti mismo } \\
\text { ¿Cuántas personas viven en } \\
\text { tu casa? }(1 ; 2 ; 3 ; 4 ; 50 \\
\text { más) }\end{array}$ & 400 & 3.66 & 1.18 & 1 & 5 \\
\hline CASAPROPIA & $\begin{array}{l}\text { ¿En qué tipo de alojamiento } \\
\text { vive usted? ( } 1 . \text { Casa propia, } \\
0 . \text { Otra) }\end{array}$ & 400 & 0.78 & 0.41 & 0 & 1 \\
\hline EDU & $\begin{array}{l}\text { ¿Cuál es el más alto grado } \\
\text { de escolaridad que tú } \\
\text { completaste? (1. Educación } \\
\text { básica; } 2 \text {. Preparatoria; } 3 \text {. } \\
\text { Licenciatura trunca; } 4 . \\
\text { Licenciatura completa; } 5 . \\
\text { Posgrado) }\end{array}$ & 400 & 2.60 & 1.27 & 0 & 5 \\
\hline
\end{tabular}


Tecnología y

Ciencias $₫$ Agua
2021, Instituto Mexicano de Tecnología del Agua

Open Access bajo la licencia CC BY-NC-SA 4.0

(https://creativecommons.org/licenses/by-nc-sa/4.0/)

\begin{tabular}{|lllllll|}
\hline \multirow{2}{*}{ INGRESO } & $\begin{array}{l}\text { ¿Cuál es el ingreso mensual } \\
\text { de tu hogar? }(1 . \$ 2,650 \text { a }\end{array}$ & & & & & \\
& $\$ 13,254 ; 2 . \$ 13,254$ a $\$$ & 381 & 1.89 & 0.79 & 1 & 4 \\
& 26,$508 ; 3 . \$ 26,508$ a & & & & & \\
& $\$ 39,662 ; 4$. Más de 39,662) & & & & & \\
\hline EDAD & Edad exacta & 400 & 45.51 & 19.09 & 18 & 57 \\
\hline
\end{tabular}

*Estas preguntas fueron diseñadas originalmente en cinco niveles, pero fueron reescaladas a tres, porque había pocas respuestas en algunos niveles. Se unieron "completamente de acuerdo" con "medianamente de acuerdo", y "totalmente en desacuerdo" con "medianamente en desacuerdo". "Ni de acuerdo ni en desacuerdo" se dejó como originalmente fue respondida.

**Esta pregunta fue reescalada de cuatro niveles a tres, porque en el nivel cuatro había muy pocas respuestas. Se fusionó "siempre" y "la mayoría del tiempo", pues ambas señalan que las autoridades responsables del agua hacen lo correcto.

*** Se unieron los niveles más altos ( 3 y 4 en 3 ) y los niveles más bajos ( 1 y 2 en 1 ).

De los encuestados, el $31 \%$ no está dispuesto a pagar más por la calidad del agua; $22 \%$ pagaría hasta 40 pesos más; $16 \%$ hasta $60 ; 19$ $\%$ hasta 100, y el restante $12 \%$ más de 100 pesos. En cuanto a las características respecto al consumo de agua, el 99 \% toma agua embotellada y el $77 \%$ estaría dispuesto a tomar agua de la llave si la calidad mejorara.

Sobre la percepción del agua suministrada, $39 \%$ considera que no tiene mal olor; mientras que el $8 \%$ es indiferente, y la gran mayoría (53 \%) percibe que el agua huele mal. El $25 \%$ de los encuestados está en desacuerdo en que el agua esté contaminada; $7 \%$ son indiferentes, y el 
69 \% piensa que sí está contaminada. La gran mayoría (59 \%) no percibe que exista escasez en su colonia; $5 \%$ son indiferentes, y $35 \%$ piensan que sí hay un problema de escasez. A casi tres cuartas partes (74\%) les preocupa enfermarse por tomar agua de la llave. Solo $21 \%$ percibe que las autoridades encargadas de la gestión del agua hacen lo correcto, mientras $54 \%$ son indiferentes y $26 \%$ no están de acuerdo.

Con respecto a las características del hogar y los entrevistados, en el $31 \%$ de las viviendas habitan más de cinco personas; en $27 \%$, cuatro; en $22 \%$, tres; en $16 \%$, dos, y en $4 \%$ solamente una. En $79 \%$ de los hogares encuestados no hay personas con problemas de salud; en $16 \%$ hay una persona enferma, y en el restante $7 \%$ más de una. El $78 \%$ de los casos respondió vivir en casa propia y el promedio de edad fue de 46 años. El $1 \%$ de los encuestados no estudió; $25 \%$ hizo educación básica; $28 \%$, preparatoria; $10 \%$, licenciatura trunca; $1 \%$, carrera técnica; 33 \% completó la licenciatura, y solo 3 \% cuenta con posgrado. Sobre el nivel de ingreso, el $39 \%$ gana menos de $\$ 13254$ pesos; $40 \%$, entre el nivel anterior y $\$ 26508$; el $19 \%$, entre $\$ 26508$ y $\$ 39662$, y sólo el 2 $\%$ ingresa más de \$39662.

Se les hizo una pregunta sobre el nivel de confianza en los demás y el $50 \%$ contestó que no se debe confiar; $34 \%$, que se debe confiar medianamente, y solo $16 \%$ respondió que sí se debe confiar.

Sobre las estadísticas antes mencionadas, podemos destacar que sí existe una percepción de que no se puede tomar agua de la llave, ya sea porque tiene mal olor o está contaminada, y se teme contraer alguna 
enfermedad por este motivo. Por lo anterior, las personas encuestadas sí estarían dispuestas a tomar del grifo si la calidad del agua mejorara. En el siguiente apartado se desarrolla un modelo econométrico, con la finalidad de encontrar los factores que se asocian con la disposición a pagar para la mejora en la calidad del agua en Zapopan, Jalisco.

\section{Modelo econométrico}

El modelo implementado es un logit ordenado, porque permite la posibilidad de observar si algunas características de los usuarios, así como sus percepciones, están asociadas con el nivel de disposición de pago para mejorar la calidad del agua. La variable dependiente del modelo es disposición a pagar (DISPAGAR). Las variables explicativas son INGRESO; EDAD; precepción de mal olor (MALOLOR) y de contaminación del agua (CONTAMINADA); la preocupación por enfermarse por tomar agua de la llave (ENFERMARSE); pensar que las autoridades responsables del agua hacen lo correcto (AUTBIEN); percepción de escasez de agua en la colonia (ESCASEZ); confianza en las demás personas (CONFIANZA); personas que viven en casa (HABITANTES); personas con mala salud en la vivienda (ENFERMOS); tipo de alojamiento medido a través de la variable 
dicotómica CASAPROPIA; la edad exacta (EDAD); el grado de escolaridad (EDU), y el nivel de ingreso mensual del hogar (INGRESO).

Los resultados del modelo logit ordenado se observan en la Tabla 3. El modelo 1 es la base; los 2 y 3 conservan las variables más significativas, y el 4 busca encontrar un resultado más parsimonioso. En general, los signos y la significancia de las variables son consistentes en todos los modelos. La pseudo- $R$ cuadrada en todos los modelos ronda el 0.08. Sin embargo, un indicador de ajuste más funcional para este tipo de modelos es el porcentaje correctamente predicho, que ronda el $37 \%$. Lo anterior muestra un buen ajuste, al considerar que son siete niveles de disposición a pagar. Y si se divide 100 \% entre siete arroja un $14 \%$. Es decir, el modelo acierta $23 \%$ más que si simplemente se eligiera la disposición a pagar aleatoriamente.

Tabla 3. Modelo logit ordenado sobre la disposición a pagar para mejorar la calidad del agua en Zapopan. Fuente: elaboración propia.

\begin{tabular}{ccccc}
\hline Variable & $\begin{array}{c}\text { (Modelo 1) } \\
\text { DISPAGAR }\end{array}$ & $\begin{array}{c}\text { (Modelo 2) } \\
\text { DISPAGAR }\end{array}$ & $\begin{array}{c}\text { (Modelo 3) } \\
\text { DISPAGAR }\end{array}$ & $\begin{array}{c}\text { (Modelo 4) } \\
\text { DISPAGAR }\end{array}$ \\
\hline EMBOTELLADA & $1.880(1.318)$ & $1.812(1.329)$ & & \\
LLAVESIMEJORA & $1.789 * * *(0.268)$ & $1.778 * * *(0.268)$ & $1.742 * * *(0.266)$ & $1.723 * * *(0.264)$ \\
MALOLOR & $-0.287 * *(0.115)$ & $-0.314 * * *(0.106)$ & $-0.272 * * *(0.105)$ & $-0.271 * * *(0.100)$ \\
CONTAMINADA & $-0.080(0.126)$ & & & \\
ENFERMARSE & $0.142(0.124)$ & $0.129(0.123)$ & & \\
AUTBIEN & $0.267 *(0.149)$ & $0.278 *(0.147)$ & $0.250 *(0.147)$ &
\end{tabular}


Tecnología y

Ciencias $₫$ Agua
2021, Instituto Mexicano de Tecnología del Agua

Open Access bajo la licencia CC BY-NC-SA 4.0

(https://creativecommons.org/licenses/by-nc-sa/4.0/)

$\begin{array}{ccccc}\text { ESCASEZ } & 0.169(0.103) & 0.166(0.103) & 0.178 *(0.103) & \\ \text { CONFIANZA } & -0.332 * *(0.132) & -0.318 * *(0.131) & -0.287 * *(0.130) & \\ \text { HABITANTES } & 0.062(0.083) & & & \\ \text { ENFERMOS } & 0.283 *(0.154) & 0.286 *(0.153) & & \\ \text { CASAPROPIA } & 0.085(0.229) & & & \\ \text { EDAD } & -0.019 * * *(0.005) & -0.020 * * *(0.005) & -0.019 * * *(0.005) & -0.019 * * *(0.005) \\ \text { EDU } & 0.009(0.089) & & & 0.382 * * *(0.124) \\ \text { INGRESO } & 0.379 * * *(0.145) & 0.396 * * *(0.125) & 0.375 * * *(0.124) & 380 \\ \text { Observaciones } & 380 & 380 & 380 & 0.07 \\ \text { Pseudo R cuadrada } & 0.08 & 0.08 & 0.08 & 36.1 \% \\ \text { PCP }{ }^{1} & 37.4 \% & 37.4 \% & 36.8 \% & \end{array}$

${ }^{1}$ Porcentaje correctamente predicho.

Notas: entre paréntesis los errores estándar; ${ }^{*},{ }^{* *},{ }^{* * *}$ son significancia al 10,5 y $1 \%$, respectivamente.

La variable LLAVESIMEJORA es estadísticamente significativa con signo positivo al $1 \%$ en todos los modelos. Lo anterior significa que si la persona está abierta a tomar agua del grifo si la calidad lo permitiera, estaría dispuesta a pagar más.

En cuanto a MALOLOR, resulta significativa negativa en todos los modelos. En tres de ellos al $1 \%$ y en uno al $5 \%$. Por tanto, la percepción de mal olor está asociada con menor disposición de pago en las personas encuestadas. Este resultado es contrastante con estudios de otros países, en los que el mal olor se asocia con mayor disposición a pagar. Quizás esto tenga que ver con que en otros países las personas confían más en 
la capacidad de las autoridades para dar respuesta a las demandas ciudadanas.

La variable AUTBIEN (percibir que las autoridades del agua hacen lo correcto) es significativa positiva al $10 \%$ en tres de los modelos. Esto implica que si las personas confían en las autoridades responsables del agua están más dispuestas a pagar por mejorar la calidad del recurso hídrico suministrado.

La variable ESCASEZ sólo resulta significativa al $10 \%$ en uno de los modelos. Lo que nos sugiere es que, ante la percepción de escasez de agua, la gente está dispuesta a pagar más por mejorar la calidad del servicio.

Confiar en las demás personas (CONFIANZA) es significativa negativa al $5 \%$ en tres de los modelos. En general, las personas con más confianza son menos propensas a estar dispuestas a pagar más por la calidad del agua. Al sentirse seguros y confortables con su entorno, tienen menos preocupación por mejorar los servicios públicos que reciben.

El número de personas con problemas de salud (ENFERMOS) resultó significativa positiva al $10 \%$ en dos de los modelos. El tener personas enfermas en casa impacta positivamente en la disposición a pagar por la calidad del agua. Lo anterior es predecible, dado que existe preocupación de que los familiares no tengan afectaciones por agua contaminada.

La variable EDAD resulta significativa negativa al $1 \%$ en todos los modelos. Personas de mayor edad son menos propensas a pagar más por la calidad del agua que los más jóvenes. $Y$ las personas con mayor 
INGRESO están dispuestas a pagar más. La variable resultó significativa positiva al $1 \%$.

Las razones de momios de los modelos logit ordenados se muestran en la Tabla 4. Aquellos coeficientes que tienen valores mayores a uno indican que cuando la variable aumenta es más probable que se incremente, en las personas encuestadas, la disposición a pagar por la calidad del agua. En cambio, cuando son menores a uno, si aumenta la variable es menos probable que las personas estén dispuestas a erogar más dinero para mejorar la calidad del recurso hídrico recibido. En los siguientes párrafos se hace la interpretación de los coeficientes de las variables que resultaron significativas.

Tabla 4. Razones de momios del modelo logit ordenado. Fuente: elaboración propia.

\begin{tabular}{ccccc}
\hline Variable & $\begin{array}{c}\text { (Modelo 1) } \\
\text { DISPAGAR }\end{array}$ & $\begin{array}{c}\text { (Modelo 2) } \\
\text { DISPAGAR }\end{array}$ & $\begin{array}{c}\text { (Modelo 3) } \\
\text { DISPAGAR }\end{array}$ & $\begin{array}{c}\text { (Modelo 4) } \\
\text { DISPAGAR }\end{array}$ \\
\hline EMBOTELLADA & $6.551(8.635)$ & $6.121(8.132)$ & & \\
LLAVESIMEJORA & $5.981 * * *(1.602)$ & $5.920 * * *(1.584)$ & $5.707 * * *(1.518)$ & $5.601 * * *(1.450)$ \\
MALOLOR & $0.751 * * *(0.086)$ & $0.730 * * *(0.078)$ & $0.762 * * *(0.080)$ & $0.762 * * *(0.076)$ \\
CONTAMINADA & $0.923(0.112)$ & & & \\
ENFERMARSE & $1.152(0.143)$ & $1.137(0.140)$ & & \\
AUTBIEN & $1.306 *(0.194)$ & $1.321 *(0.195)$ & $1.284 *(0.189)$ & \\
ESCASEZ & $1.184(0.122)$ & $1.181(0.121)$ & $1.195 *(0.123)$ & \\
CONFIANZA & $0.717 * *(0.095)$ & $0.728 * *(0.095)$ & $0.750 * *(0.098)$ & \\
HABITANTES & $1.064(0.088)$ & & &
\end{tabular}


Tecnología y

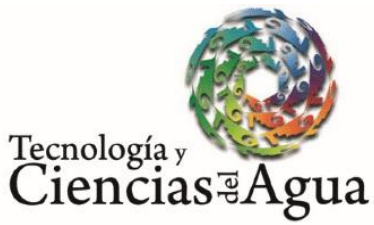

2021, Instituto Mexicano de Tecnología del Agua

Open Access bajo la licencia CC BY-NC-SA 4.0

(https://creativecommons.org/licenses/by-nc-sa/4.0/)

$\begin{array}{ccccc}\text { ENFERMOS } & 1.328 *(0.205) & 1.331^{*}(0.203) & & \\ \text { CASAPROPIA } & 1.089(0.250) & & & \\ \text { EDAD } & 0.981 * * *(0.005) & 0.980 * * *(0.005) & 0.981 * * *(0.005) & 0.981 * * *(0.005) \\ \text { EDU } & 1.009(0.089) & & & \\ \text { INGRESO } & 1.461 * * *(0.212) & 1.486 * *(0.185) & 1.455 * * *(0.181) & 1.465 * * *(0.181) \\ \text { Observaciones } & 380 & 380 & 380 & 380 \\ \text { Pseudo R cuadrada } & 0.08 & 0.08 & 0.08 & 0.07 \\ \text { PCP } & 37.4 \% & 37.4 \% & 36.8 \% & 36.1 \%\end{array}$

${ }^{1}$ Porcentaje correctamente predicho.

Notas: entre paréntesis los errores estándar; $* * *, * * *$ son significancia al $10 \%, 5 \%$ y $1 \%$ respectivamente.

Por un incremento de una unidad en la variable LLAVESIMEROJA, los momios de alta disposición a pagar contra la combinación de media y baja disposición a pagar incrementan entre 5.6 y 5.9 veces, dejando las otras variables sin cambio en el modelo.

Si se da un incremento de una unidad en la variable MALOLOR, los momios de alta disposición a pagar contra la combinación de media y baja disposición a pagar son entre 0.73 y 0.62 veces menores, dejando las otras variables sin cambio en el modelo.

Ante un incremento en la variable AUTBIEN, los momios de alta disposición a pagar contra la combinación de media y baja disposición a pagar incrementan entre 1.2 y 1.3 veces, dejando las otras variables sin cambio en el modelo. 
Si existe un incremento en la variable ESCASEZ, los momios de alta disposición a pagar contra la combinación de media y baja disposición a pagar incrementan 1.19 veces, lo cual supone ausencia de cambios en las otras variables del modelo.

Por un incremento de una unidad en la variable CONFIANZA, los momios de alta disposición a pagar contra la combinación de media y baja disposición a pagar son entre 0.71 y 0.75 veces menores, dejando las otras variables sin cambio en el modelo.

Ante un incremento de una unidad en la variable ENFERMOS, los momios de alta disposición a pagar contra la combinación de media y baja disposición a pagar incrementan 1.3 veces, dejando las otras variables sin cambio en el modelo.

Por un incremento de una unidad en la variable EDAD, los momios de alta disposición a pagar contra la combinación de media y baja disposición a pagar son 0.98 veces menores, dejando las otras variables sin cambio en el modelo.

Si existe un incremento en la variable INGRESO, los momios de alta disposición a pagar contra la combinación de media y baja disposición a pagar incrementan 1.4 veces, lo cual supone ausencia de cambios en las otras variables del modelo. 


\section{Discusión y conclusiones}

La encuesta realizada a los 400 hogares representativos de Zapopan arrojó que los consultados toman agua embotellada (99\%); consideran que el agua suministrada huele mal (53\%) y que está contaminada (69 $\%)$; temen por su salud o la de sus familiares por beber del grifo (74 \%), pero estarían dispuestos a tomar agua del mismo si la calidad mejorara (77 \%). Sin embargo, más de la mitad no estaría dispuesta a pagar más por mejorar la calidad del agua o pagaría no más de 40 pesos (31 y 22 $\%$, respectivamente).

Como se aprecia en los modelos expuestos en el presente artículo, la disposición a pagar por incrementar la calidad del agua en Zapopan está asociada significativa y positivamente con estarabierto a tomar agua de la llave si se logra este objetivo (LLAVESIMEJORA); con percibir que las autoridades encargadas del agua actúan bien (AUTBIEN); con una creencia de escasez de agua en la colonia (ESCASEZ); con tener personas con problemas de salud en casa (ENFERMOS), y con el nivel de ingresos. Por otro lado, la disposición de pago se relaciona negativa y significativamente con la percepción de que el agua huela mal (MALOLOR), el nivel de confianza en los otros (CONFIANZA) y la edad.

Hay factores que son consistentes con la literatura, como el caso de la relación negativa de la disposición a pagar con la edad (Vásquez et al. 
2009; Chatterjee et al., 2017), y la relación positiva con el nivel de ingresos si fuera medido a través del nivel de educación (Chatterjee et al., 2017). En cuanto a la significancia positiva de la variable ingreso con la disposición de pago, este estudio coincide con los realizados para los casos de la ciudad griega de Mitilene (Polyzou et al., 2011), y Parral, Chihuahua, en México (Vásquez et al., 2009). Sin embargo, en otras variables no hay consistencia.

En el caso del estudio de Jacksonville, en el que se pregunta si hay preocupación por contraer enfermedades al beber agua contaminada, dicha variable resultó ser significativa positiva de la disposición a pagar por mejorar la calidad del agua. En el caso de Zapopan no resultó significativa la preocupación por contraer enfermedades, pero sí el número de personas con problemas de salud en casa. De igual forma, ambos estudios nos revelan que es relevante la disposición a pagar por el tema de salud.

Con respecto a que el agua huele mal, en la investigación en Jacksonville hay una asociación positiva entre el mal olor y la disponibilidad a pagar. En Zapopan resultó ser diferente. Ante incrementos en la percepción de mal olor, la gente está menos dispuesta a pagar. Estas diferencias podrían explicarse por los contextos de las ciudades. Las personas de la ciudad estadounidense podrían confiar más en la capacidad de sus gobiernos para atender demandas ciudadanas que en el caso del municipio mexicano. 
Sobre la pregunta acerca de la confianza en el gobierno hubo diferencias en la manera de cuestionar. En el estudio de la ciudad estadounidense, a mayor desconfianza promedio en las autoridades hay menor disposición a pagar por la mejora en la calidad del agua. En la investigación de Zapopan se preguntó si las autoridades encargadas del agua actuaban de manera correcta, resultando dicha variable significativa positiva. Es decir, aunque se preguntó de diferente manera, la conclusión en este punto es similar. Cuando hay confianza en la autoridad o se percibe que hace bien su trabajo, la disposición a pagar aumenta.

En la investigación en Zapopan se agregó la variable confianza en otros, misma que resultó significativa negativa. Esto es un hallazgo interesante, pues nos revela que cuando existe un nivel de confianza social alto, las preocupaciones por el incremento en la calidad de los servicios públicos disminuyen. Quizás estos resultados no serían consistentes si en vez de hablar de incrementar la calidad del agua, se hablara de la provisión del servicio en casos donde hubiera tandeos o falta de servicio continuo.

En este estudio también se incluye una pregunta sobre la percepción de escasez de agua, resultando ligeramente significativa en uno de los modelos. Sería interesante explorar esta variable en contextos donde existan problemas más serios de falta de agua.

Dado las evidencias antes mencionadas, resulta relevante señalar que sí hay disposición a pagar más por tomar agua de la llave si hay una percepción de que el servicio mejora, situación consistente con la negativa 
a pagar más si el agua huele mal. Asimismo, aquellos hogares con más ingreso muestran mayor disposición a pagar para una mejor calidad del agua. Ante lo anterior, para la autoridad podría ser factible ajustar al alza la tarifa de aquellos estratos de alto ingreso y así poder hacerse de recursos para mejorar la calidad del agua en la urbe. Como lo sugieren los modelos, es muy importante que dichos recursos se enfoquen en evitar el mal olor del líquido. Y, una vez mejorado el servicio, es de vital importancia informarlo a la comunidad para cambiar la percepción en sentido positivo. Sin embargo, antes de exigir incrementos de tarifas a los usuarios, el organismo operador de agua debe hacer un compromiso por incrementar su eficiencia operativa y comercial, así como ser transparente en el uso de los recursos.

En conclusión, este estudio nos muestra evidencia de que cuando se percibe que existe un buen trabajo de la autoridad, la gente está dispuesta a pagar más por mejorar la calidad de los servicios públicos. Asimismo, se demuestra que cuando se hace la misma pregunta en contextos distintos, la significancia de las variables puede ser contrastante, como en el caso de la percepción del mal olor. Como futuros posibles estudios sería interesante explorar la disposición a pagar por el suministro continuo de agua en lugares donde existen problemas de abastecimiento; y seguir explorando las preocupaciones concretas en materia de salud, al ser una variable que en lo general ha sido significativa. 


\section{Agradecimientos}

Esta investigación fue elaborada con el apoyo financiero de la Universidad Panamericana a través del "Fondo fomento a la Investigación UP2018" (Código del proyecto UP-CI-2018-EMP-GDL-01).

\section{Referencias}

Bateman, I., Carson, R., Day, B., Hanemann, M., Hanley, N., Hett, T., Jones-Lee, M., \& Loomes, G. (2002). Economic Valuation with Stated Preference Techniques. UK: Edward Elgar Publishing Limited. Recuperado de https://doi.org/10.4337/9781781009727

Briseño, H., \& Sánchez, A. (2018). Decentralization, consolidation, and crisis of urban water management in Mexico. Tecnología y ciencias del agua, 9(4), 25-47. Recuperado de https://doi.org/10.24850/j-tyca2018-04-02

Cameron, T., \& James, M. (1987). Efficient estimation methods for "closed-ended" contingent valuation surveys. The Review of Economics and Statistics, 69(2), 269-276. DOI : 10.2307/1927234

Camps, S. (2017). La hidrosensibilidad como propuesta para la solución de la crisis del agua en el entorno urbano: el caso de la Zona Metropolitana de Guadalajara. Equilibrio Económico, Revista de Economía, Política y Sociedad, 13(2), 191-215.

Chatterjee, C., Triplett, R., Johnson, C. K., \& Ahmed, P. (2017). Willingness to pay for safe drinking water: A contingent valuation 
study in Jacksonville, FL. Journal of Environmental Management, 203, 413-421. Recuperado

de https://doi.org/10.1016/j.jenvman.2017.08.008

CEA, Comisión Estatal del Agua de Jalisco. (2014). Proyecto integral de sanemiento y abastecimiento de la zona conurbada de Guadalajara. Recuperado de http://www.ceajalisco.gob.mx/zcg-proyecto.swf

Conagua, Comisión Nacional del Agua. (2016). Situación del subsector agua potable, drenaje y saneamiento, edición 2016. Ciudad de México, México: Secretaría de Agricultura y Desarrollo Rural.

Friedler, E., \& Hadari, M. (2006). Economic feasibility of on-site greywater reuse in multi-storey buildings. Desalination, 190, 221-234. Recuperado de https://doi.org/10.1016/j.desal.2005.10.007

Genius, M., \& Tsagarakis, K. P. (2006). Water shortages and implied water quality: A contingent valuation study. Water Resources Research, 42. Recuperado de https://doi.org/10.1029/2005WR004833

Pattanayak, S. K., Yang, J.-C., Whittington, D., \& Bal-Kumar, K. C. (2005). Coping with unreliable public water supplies: Averting expenditures by households in Kathmandu, Nepal. Water Resources Research, 41. Recuperado de https://doi.org/10.1029/2003WR002443

Polyzou, E., Jones, N., Evangelinos, K. I., \& Halvadakis, C. P. (2011). Willingness to pay for drinking water quality improvement and the 
influence of social capital. Journal of Socio-Economics, 40(1), 74-80. Recuperado de https://doi.org/10.1016/j.socec.2010.06.010

Ramírez, J. (2018). Crisis del agua: en Monterrey, Guadalajara, San Luis Potosí, León y la Ciudad de México (1950-2010). Ciudad de México, México: Universidad Nacional Autónoma de México.

Rosado, M. A., Cunha-E-Sá, M. A., Ducla-Soares, M. M., \& Nunes, L. C. (2006). Combining averting behavior and contingent valuation data: An application to drinking water treatment in Brazil. Environment and Development Economics, 11(6), 729-746. Recuperado dedoi:10.1017/S1355770X0600324X

Smith, R. D. (2006). It's not just what you do, it's the way that you do it: The effect of different payment card formats and survey administration on willingness to pay for health gain. Health Economics, 15, 281-293. DOI: 10.1002/hec.1055

Tanellari, E., Bosch, D., Boyle, K., \& Mykerezi, E. (2015). On consumers' attitudes and willingness to pay for improved drinking water quality and infrastructure. Water Resources Research, 51, 47-57. DOI: 10.1002/2013WR014934

Torres-Rodríguez, A. (2013). Abastecimiento de agua potable en las ciudades de México: el caso de estudio de la Zona Metropolitana de Guadalajara. Agua y Territorio, 1, 77-90. DOI: 10.17561/at.v1i1.1035.

Tussupova, K., Berndtsson, R., Bramryd, T., \& Beisenova, R. (2015). 
Investigating willingness to pay to improve water supply services: application of contingent valuation method. Water, 7, 3024-3039. Recuperado de https://doi.org/10.3390/w7063024

Vásquez, W. F., Mozumder, P., Hernández-Arce, J., \& Berrens, R. P. (2009). Willingness to pay for safe drinking water: Evidence from Parral, Mexico. Journal of Environmental Management, 90(11), 33913400. Recuperado

de

https://doi.org/10.1016/j.jenvman.2009.05.009

Whittington, D., Briscoe, J., Xinming, M., \& Barron, W. (1990). Estimating the willingness to pay for water services in developing countries: A case study of the use of contingent valuation surveys in southem Haiti. Economic Development \& Cultural Change. Recuperado de https://doi.org/10.1086/451794

Whittington, D. (2002). Improving the performance of contingent valuation studies in developing countries. Environmental and Resource Economics, 22, 323-367. Recuperado de https://doi.org/10.1023/A:1015575517927 